\title{
Orquiectomía parcial en un paciente con tumor de células de Leydig asociado con síndrome de Klinefelter
}

Cumming A, ${ }^{1}$ Manzanilla-García HA, ${ }^{1}$ Venegas-Vega $C,{ }^{2}$ Arana-Trejo RM, ${ }^{2}$ Aizpuru $E{ }^{3}$ y colaboradores.

\section{Resumen}

ANTECEDENTES: el cáncer de testículo es la neoplasia más frecuente en la edad reproductiva. El 95\% de los tumores corresponden a células germinales y el $5 \%$ restante a células estromales. Representa de $1-1.5 \%$ de las neoplasias en los hombres. Los factores de riesgo más importantes son: criptorquidia, antecedentes familiares de cáncer de testículo, atrofia testicular, coexistencia de tumor contralateral, síndrome de Klinefelter y carcinoma in situ. Los tumores de células de Leydig suelen ser benignos en los niños y potencialmente malignos en 10 a $15 \%$ de los adultos.

CASO CLÍNICO: paciente masculino de 46 años de edad, que acudió a valoración médica por masa testicular. Se sometió a estudios de imagen para descartar alguna neoplasia testicular y determinación de marcadores tumorales. A la palpación del testículo izquierdo se percibió indurado, por lo que se decidió efectuar orquiectomía parcial; el estudio histopatológico reportó tumor de células de Leydig. Debido a las características fenotípicas y el resultado histopatológico se efectuó un estudio genético por sospecha de síndrome de Klinefelter. El muestreo de sangre periférica comprobó el diagnóstico de tumor de células de Leydig asociado con síndrome de Klinefelter.

CONCLUSIÓN: el riesgo de tumor de células de Leydig es mayor en pacientes con síndrome de Klinefelter que en la población general. La relación entre el tumor de células de Leydig y síndrome de Klinefelter se ha descrito en la bibliografía, pero se desconoce su fisiopatología exacta. Se requieren estudios adicionales para determinar su asociación.

PALABRAS CLAVES: síndrome de Klinefelter, tumor de células de Leydig, tumor testicular.
${ }^{1}$ Servicio de Urología, Hospital General de México Dr. Eduardo Liceaga, Ciudad de México.

${ }^{2}$ Servicio de Genética, Hospital General de México Dr. Eduardo Liceaga, Ciudad de México.

${ }^{3}$ Servicio de Genética, Hospital Ángeles del Pedregal, Ciudad de México.

Recibido: diciembre 2016

Aceptado: junio 2017

Correspondencia

Dr. Alejandro Cumming Martínez Báez

cummingmd@me.com

Este artículo debe citarse como

Cumming A, Manzanilla-García HA, Venegas-Vega C, y col. Orquiectomía parcial en un paciente con tumor de células de Leydig asociado con síndrome de Klinefelter. Rev Mex Urol. 2017 jul-agos;77(4):296-301. DOI: https://doi.org/10.24245/revmexurol.v77i4.1096 


\section{Partial orchiectomy in a case of Leydig cell tumor associated with Klinefelter syndrome}

\author{
Cumming A, ${ }^{1}$ Manzanilla-García HA, ${ }^{1}$ Venegas-Vega $C,{ }^{2}$ Arana-Trejo RM, ${ }^{2}$ \\ Aizpuru $E,{ }^{3}$ et al.
}

\begin{abstract}
BACKGROUND: Testicular cancer is the most frequent tumor in the reproductive age of the individual, representing $1-5 \%$ of neoplasias in men. Ninety-five percent of the tumors are germ cell tumors and the other $5 \%$ are of stromal origin. The most important risk factors are: cryptorchidism, a family history of testicular cancer, testicular atrophy, the presence of a contralateral tumor, Klinefelter syndrome, and carcinoma in situ. Leydig cell tumors are usually benign in children and potentially malignant in $10-15 \%$ of adult cases.
\end{abstract}

CLINICAL CASE: A 46-year-old man sought medical attention for a testicular mass and was evaluated through imaging studies and tumor markers to rule out testicular neoplasia. Upon palpation, the left testis was indurated and so partial orchiectomy was performed. The histopathologic report stated Leydig cell tumor. Given the patient's phenotypic characteristics and the histopathologic result, Klinefelter syndrome was suspected, and the patient was assessed by the genetics team. Leydig cell tumor associated with Klinefelter syndrome was corroborated through peripheral blood sampling.

CONCLUSION: The risk for presenting with Leydig cell tumor is higher in patients with Klinefelter syndrome than in the general population. The association between Leydig cell tumor and Klinefelter syndrome is described in the literature, but the precise pathophysiology is not known. Further studies are required to determine their association.

KEYWORDS: Klinefelter syndrome; Leydig cell tumor; Testicular tumor
${ }^{1}$ Urology Service, Hospital General de México, Dr. Eduardo Liceaga, Mexico City. 2 Genetics Service. Hospital General de México, Dr. Eduardo Liceaga, Mexico City.

${ }^{3}$ Genetics Service, Hospital Ángeles del Pedregal, Mexico City.

\section{Correspondence}

Dr. Alejandro Cumming Martínez Báez cummingmd@me.com

\section{ANTECEDENTES}

El cáncer de testículo se divide en diversos subtipos, de los que $95 \%$ se relacionan con tumores de células germinales. En Estados Unidos, el cáncer testicular representa 1 a $2 \%$ de las neoplasias en hombres, con incidencia de 5 casos por cada 100,000 habitantes. En ese país, supone la neoplasia más frecuente en hombres de 20 a 40 años de edad, seguida de la leucemia en sujetos de 15-19 años de edad. Con el advenimiento de la terapia con cisplatino aumentó la supervivencia libre de enfermedad entre $80-90 \%$, incluso en pacientes con metástasis.

En México, el cáncer se ha convertido en un problema de salud pública, con 110,000 casos nuevos registrados al año. Los tumores malignos 
suponen el tercer lugar de las causas de muerte en el país; en el año 2006 fallecieron 63,888 sujetos por esta causa, lo que representó $12.9 \%$ del total de las defunciones registradas en ese año. En el grupo de varones de 25 a 34 años de edad, la leucemia (18.7\%) y el tumor maligno de testículo (13.3\%) ocasionan 32 de cada 100 decesos. ${ }^{1}$

Los tumores de células germinales y los del estroma del cordón sexual constituyen los 2 tipos más predominantes. Los tumores de células de Leydig, o leydigiomas, representan 0.8-3\% de todas las neoplasias testiculares en la edad adulta y $4-9 \%$ en la infancia. La mayor parte de estos tumores son benignos, especialmente durante la infancia. Las neoplasias testiculares suelen relacionarse con testículos no descendidos, síndrome de disgenesia testicular e infertilidad. La transformación maligna ocurre en 3.5 a $14.5 \%$ de los pacientes con testículos no descendidos. ${ }^{2}$

Los testículos no descendidos, así como los ectópicos, suelen asociarse con aneuploidias en $14-28 \%$ de los casos, esto supone un valor 6 veces más elevado que en la población general. El pico de edad y la distribución histológica de los tumores en testículos no descendidos son similares a los de pacientes con testículos escrotales. Los estados hiper e hipoandrogénicos son los factores de riesgo más aceptados para padecer cáncer testicular e infertilidad. Aunque la hormona luteinizante tiene función importante en la proliferación de las células de Leydig, su maduración y desarrollo se afectan por señalizaciones paracrinas y endocrinas, incluidas las hormonas antimülleriana, inhibina y otros factores de crecimiento.

Los tumores de células de Leydig, la hiperplasia, o ambos, son alteraciones frecuentes en los exámenes histológicos de biopsias testiculares, especialmente de pacientes con atrofia testicular, criptorquidia, síndrome de Klinefelter y síndrome de insensibilidad a los andrógenos. ${ }^{2}$

El síndrome de Klinefelter afecta a 1 de cada 500 neonatos y representa la alteración genética más frecuente en los hombres, que subsecuentemente resulta en infertilidad. La mayoría de estos pacientes expresa el cariotipo $47, \mathrm{XXY}$, incluso tienen 50 veces más riesgo de padecer tumores de células germinales extragonadales. ${ }^{3}$

El síndrome de Klinefelter es la aneuploidia más común. Afecta a 1 de cada 650 recién nacidos ( $0.2 \%$ de la población general). Así mismo, existen formas más severas de aneuploidias, aunque afortunadamente menos frecuentes, como: $48, X X X Y, 48, X X Y Y$ y 49,XXXXY. Se piensa que el cariotipo 49,XXXXY surge de la no disyunción por línea materna durante ambas etapas de la meiosis, reteniendo todos los cromosomas $\mathrm{X}$ en el ovocito. Las principales alteraciones endocrinas de las aneuploidias son el hipogonadismo hipergonadotrópico, los cambios testiculares degenerativos y la formación de tumores. Los tumores de células de Leydig aparecen ocasionalmente en pacientes con síndrome de Klinefelter. ${ }^{3}$

El diagnóstico se establece por alteraciones en el desarrollo en niños y por infertilidad en adultos. La variedad 49,XXXXY es la forma más severa de la enfermedad y aparece en 1.4 a $1.7 \%$ de las aneuploidias, con la manifestación clásica de retraso mental, hipogonadismo y sinostosis radioulnar.

En la adultez temprana, los pacientes con síndrome de Klinefelter parecen entrar de forma normal a la pubertad, con desarrollo incompleto de los caracteres sexuales secundarios, pero esta manifestación es rara en los casos cuyas variantes se caracterizan por ausencia de pubertad. Los perfiles endocrinológicos en pacientes con síndrome de Klinefelter y sus variantes se distinguen por 
concentraciones normales de gonadotropinas, hormona antimülleriana e inhibina $\mathrm{B}$, además de concentraciones variables de testosterona durante la infancia. En los niños las células de Leydig suelen ser sensibles al efecto proliferativo de la hormona luteinizante y la sensibilidad de las células de Sertoli aún se discute; sin embargo, después de la pubertad, es evidente la resistencia a la hormona foliculoestimulante por estas células. El hipogonadismo es más severo en los pacientes con alguna de las otras variantes versus la forma clásica del síndrome $(47, X X Y)$. Esto se debe a un daño testicular mayor, asociado con concentraciones menores de testosterona, incluso durante la infancia, lo que resulta en genitales ambiguos. ${ }^{3}$

Los tumores de células de Leydig representan de $0.8-3 \%$ de las neoplasias testiculares. La mayor parte son benignos (se ha estimado que solo $10 \%$ son malignos). Al igual que en el resto de los tumores testiculares, su manifestación se caracteriza por una masa indolora; algunos pacientes pueden expresar signos de feminización, como ginecomastia, disfunción eréctil y pérdida de la libido. ${ }^{4}$

La orquiectomía está indicada en pacientes con tumores de células de Leydig, ya sean únicos, unilaterales, bien circunscritos, hipervascularizados, con necrosis, litiasis o calcificación detectada en el ultrasonido.

A continuación se expone un caso de tumor de células de Leydig asociado con síndrome de Klinefelter, en un paciente atendido en el Hospital General de México Dr. Eduardo Liceaga.

\section{CASO CLÍNICO}

Paciente masculino de 46 años de edad, con antecedentes patológicos de criptorquidia derecha (tratado mediante orquidopexia a los 12 años de edad), hidrocele derecho (intervenido por hidrocelectomía a los 40 años de edad), ginecomastia con aparición hace 19 años y tratada mediante mastectomía hace 18, litiasis ureteral izquierda (tratada con ureteroscopia en 2016) y diagnóstico de infertilidad. El paciente acudió por primera vez al servicio de Urología por un cuadro de litiasis ureteral izquierda, diagnosticado mediante tomografía computada, en la que se encontró un testículo con varias zonas con heterogeneidad en sus densidades, por lo que se protocolizó para descartar alguna neoplasia testicular; sin embargo, el ultrasonido también mostró esa característica. La determinación de marcadores tumorales se encontró dentro de los parámetros de referencia. La exploración del testículo izquierdo reportó: dimensiones de $2 \mathrm{x}$ $2 \mathrm{~cm}$, con masa delimitada pétrea de aproximadamente $1 \mathrm{~cm}$, por lo que se decidió efectuar orquiectomía parcial, el informe histopatológico fue de tumor de células de Leydig (Figuras $\mathbf{1}$ y 2). De acuerdo con las características fenotípicas y el resultado histopatológico, se realizó el estudio genético para determinar el cariotipo, debido a la sospecha de síndrome de Klinefelter (Figura 3). Posteriormente se realizaron estudios complementarios de perfil hormonal y espermatobioscopia, que confirmaron el hipogonadismo hipergonadotrópico y la azoospermia. Por su parte, el cariotipo también confirmó el diagnóstico de síndrome de Klinefelter. Después de 6 meses de la intervención quirúrgica se encontró al paciente con adecuada evolución, sin evidencia de recurrencia bioquímica ni clínica del tumor.

\section{DISCUSIÓN}

El síndrome de Klinefelter es la aneuploidia más común. Afecta a 1 de cada 650 recién nacidos ( $0.2 \%$ de la población general). Las principales alteraciones endocrinas de las aneuploidias son el hipogonadismo hipergonadotrópico, los cambios testiculares degenerativos y la formación de tumores, ${ }^{5}$ como se observó en el caso aquí reportado, que nos llevó a la sospecha clínica. 


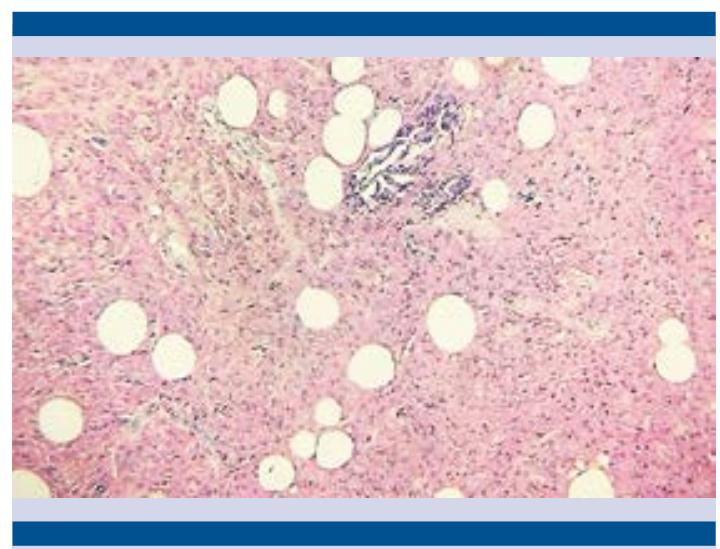

Figura 1. Tumor de células de Leydig (10x).

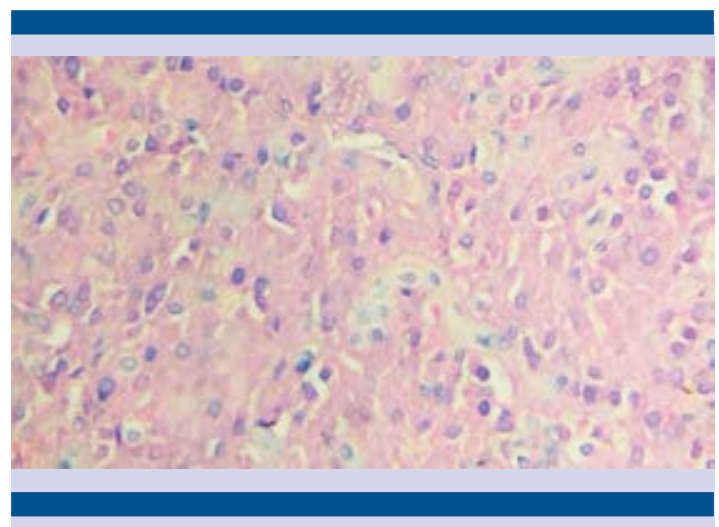

Figura 2. Tumor de células de Leydig (40x).

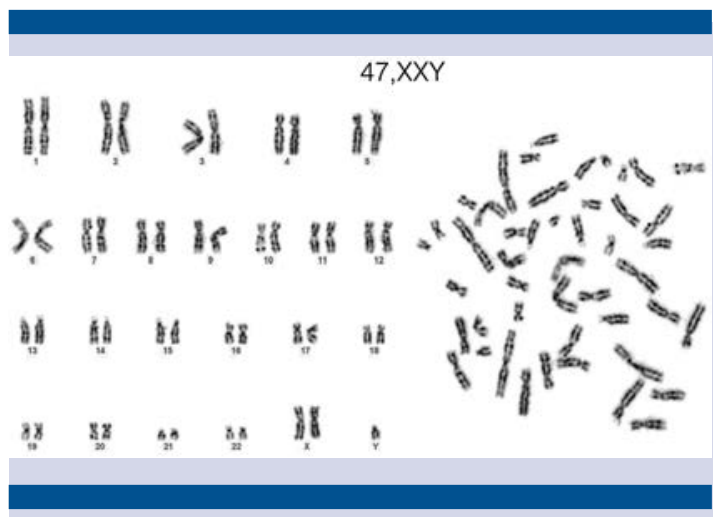

Figura 3. Expresión del cariotipo.
El síndrome de Klinefelter es causa común de hipogonadismo hipergonadotrópico, debido a concentraciones anormales de la hormona foliculoestimulante y la hormona luteinizante circulantes. Los pacientes tienen cifras bajas de testosterona y diferentes características clínicas: desde testículos pequeños y firmes, hasta la posibilidad de ginecomastia y conteo espermático bajo. ${ }^{6,7}$

Los tumores de células de Leydig, o leydigiomas, aparecen ocasionalmente en pacientes con síndrome de Klinefelter. El subtipo más frecuente en estos casos es el de células germinales..$^{8,9}$

La fisiopatología más aceptada de esta enfermedad es que el cáncer procede de un estado hiperestrogénico e hipoandrogénico, que supone cierto grado de infertilidad y daño crónico en los túbulos seminíferos y las células de Leydig. ${ }^{9}$ Sin embargo, estas últimas suelen afectarse por la acción de la hormona luteinizante, aunque se ha demostrado la participación de la inhibina B y diversas hormonas antimüllerianas. ${ }^{10}$

Butruille y su grupo describieron 45 tumores en pacientes infértiles, de los cuales 11 tenían hiperplasia de células de Leydig, 17 tumores benignos, 10 síndrome de Klinefelter y 12 tumores de células de Leydig, todos de carácter benigno. ${ }^{11}$ Debido a la gran cantidad de resultados histopatológicos que demuestran el carácter benigno en pacientes con diagnóstico de síndrome de Klinefelter, decidimos realizar orquiectomía parcial, obteniendo un resultado benigno en el estudio histopatológico.

Otros reportes describen casos aislados de Leydigiomas en pacientes con síndrome de Klinefelter, lo que coincide que son raros en este tipo de patología subyacente; sin embargo, en algunos casos puede estar claramente subdiagnosticado. ${ }^{12}$ 
El riesgo de tumor de células de Leydig en pacientes con aneuploidias es una asociación real. La incidencia es similar a la estimada en la población general, aunque algunos casos pueden ser subdiagnosticados. Deben solicitarse estudios genéticos en los pacientes con diagnóstico de tumor de células de Leydig. El tratamiento de elección consiste en orquiectomía radical, incluso la orquiectomía parcial es una buena opción, dada la evolución benigna en la mayoría de los pacientes.

\section{REFERENCIAS}

1. Flores-Novelo G, Huerta-Gómez JC, Saucedo-Salinas LA, et al. Diagnóstico y tratamiento del tumor maligno de testículo en todas las edades. Guía de Práctica Clínica del Sistema Nacional de Salud. 2014;1-45.

2. Maqdasy S, Bogenmann L, Batisse-Lignier M, et al. Leydig cell tumor in a patient with 49,XXXXY karyotype: a review of literature. Reprod Biol Endocrinol 2015;13:72.

3. Shaw NM, Stauffer C, Eisenberg ML. Leydig cell tumor found incidentally during microscopic testicular sperm extraction in patient with mosaic Klinefelter syndrome: case report. Fertil Steril 2016;106(6):1344-1347.
4. Tazi MF, Mellas S, EL Fassi MJ et al. Leydig cell hyperplasia revealed by gynecomastia. Rev Urol 2008;10:164-7.

5. Maqdasy S, Bogenmann L, Batisse-Lignier M, et al. Leydig cell tumor in a patient with 49,XXXXY karyotype: a review of literature. Reprod Biol Endocrinol 2015;13:72.

6. Wang C, Baker HW, Burger HG, et al. Hormonal studies in Klinefelter's syndrome. Clin Endocrinol (Oxf) 1975;4:399.

7. Bojesen A, Juul S, Gravholt $\mathrm{CH}$. Prenatal and postnatal prevalence of Klinefelter syndrome: a national registry study. J Clin Endocrinol Metab 2003;88:622.

8. Dilworth JP, Farrow GM, Oesterling JE. Non-germ cell tumors of testis. Urology 1991;37:399.

9. Völkl TM, Langer T, Aigner T, et al. Klinefelter syndrome and mediastinal germ cell tumors. Am J Med Genet A 2006;140:471.

10. Liu G, Duranteau L, Carel JC, Monroe J, Doyle DA, Shenker A. Leydig-cell tumors caused by an activating mutation of the gene encoding the luteinizing hormone receptor. $\mathrm{N}$ Engl J Med. 1999;341:1731-6.

11. Butruille C, Marcelli F, Ghoneim T, Lemaitre L, Puech P, Leroy $X$, et al. Prise en charge des nodules testiculaires dans une population de patients infertiles. Prog En Uro. 2012;22:45-52.

12. Fishman MDC, Eisenberg DA, Horrow MM. Klinefelter syndrome with leydig cell tumor/hyperplasia. Ultrasound Q. 2010;26:101-2.

\section{AVISO PARA LOS AUTORES}

Revista Mexicana de Urología tiene una nueva plataforma de gestión para envío de artículos: https://www.revisionporpares.com/index.php/RMUrol ahí podrá inscribirse a la base de datos administrada por el sistema Open Journal System (OJS) que ofrece las siguientes ventajas para los autores:

- Subir sus artículos directamente al sistema.

- Conocer, en cualquier momento, el estado de los artículos enviados, es decir, si ya fueron asignados a un revisor, aceptados con o sin cambios, o rechazados.

- Participar en el proceso editorial corrigiendo y modificando sus artículos hasta su aceptación final. 